\title{
Depressive Symptoms in Multiple Sclerosis from an In Vivo Study with TBSS
}

\author{
Yujuan Shen, ${ }^{1}$ Lijun Bai, ${ }^{2}$ Ying Gao, ${ }^{1}$ Fangyuan Cui, ${ }^{1}$ Zhongjian Tan, ${ }^{3}$ \\ Yin Tao, ${ }^{2}$ Chuanzhu Sun, ${ }^{2}$ and Li Zhou ${ }^{1}$ \\ ${ }^{1}$ Department of Neurology, Dongzhimen Hospital Affiliated to Beijing University of Chinese Medicine, Beijing 100700, China \\ ${ }^{2}$ The Key Laboratory of Biomedical Information Engineering, Ministry of Education, Department of Biomedical Engineering, \\ School of Life Science and Technology, Xian Jiaotong University, Xian 710049, China \\ ${ }^{3}$ Department of MRI Scanning, Dongzhimen Hospital Affiliated to Beijing University of Chinese Medicine, Beijing 100700, China
}

Correspondence should be addressed to Lijun Bai; bailj4152615@gmail.com and Li Zhou; zhouljk7211@163.com

Received 21 February 2014; Revised 5 April 2014; Accepted 8 April 2014; Published 4 May 2014

Academic Editor: Lin Ai

Copyright (C) 2014 Yujuan Shen et al. This is an open access article distributed under the Creative Commons Attribution License, which permits unrestricted use, distribution, and reproduction in any medium, provided the original work is properly cited.

\begin{abstract}
Clinically significant depression can impact up to $50 \%$ of patients with multiple sclerosis (MS) over a course of their life time, which is associated with an increased morbidity and mortality. In our study, fifteen relapsing-remitting MS (RRMS) patients and 15 age- and gender-matched normal controls were included. Diffusion tensor imaging (DTI) was acquired by employing a single-shot echo planar imaging sequence on a 3.0 T MR scanner and fractional anisotropy (FA) was performed with tract-based spatial statistics (TBSS) approach. Finally, widespread WM and GM abnormalities were observed in RRMS patients. Moreover, the relationships between the depressive symptoms which can be measured by Hamilton depression rating scale (HAMD) as well as clinical disabilities measured by the expanded disability status scale (EDSS) and FA changes were listed. There was a positive relation between EDSS and the FA changes in the right inferior parietal lobule, while negative relation was located in the left anterior cingulate cortex and hippocampus. Also a positive relation between HAMD and FA changes was found in the right posterior middle cingulate gyrus, the right hippocampus, the left hypothalamus, the right precentral gyrus, and the posterior cingulate which demonstrated a link between the depressive symptoms and clinically relevant brain areas in RRMS patients.
\end{abstract}

\section{Introduction}

Multiple sclerosis (MS) is an autoimmune disease of the central nervous system (CNS) characterized by inflammation, demyelination, and neurodegeneration [1] and it is the most common cause of neurological disability among young adults, affecting approximately one in 1,000 individuals in Europe and North America [2]. Affective disorders are common and disabling conditions in MS. Clinically significant depression can affect up to $50 \%$ of patients with MS over the course of their lifetime and it is associated with an increased morbidity and mortality [3]. Also disability is a common symptom in MS patients [4]. The factors responsible for mood disturbances in MS are still controversial: a psychological reaction to a progressively disabling and unpredictable disease may be a relevant contributor while reactive mechanisms alone are probably not sufficient to account for the high prevalence and wide spectrum of depression.

Brain magnetic resonance imaging (MRI) lesions are highly associated with depression in MS $[5,6]$. Neuroimaging studies in patients with MS have revealed associations between brain abnormalities and depression. The study by Pujol et al. [7] using axial spin echo sequence investigated three major anatomic divisions (basal, medial, and lateral) of the frontotemporal WM. They found that depressive score was significantly correlated with lesion load (LL) of the left accurate fasciculus region; in particular lesions in this area accounted for $17 \%$ of depressive score variance. Another MRI study including 95 consecutive MS patients, of which 19\% of the patients met the criteria for depression, reported that the severity of depression was correlated with right frontal 
lobule and with right temporal brain atrophy; furthermore, T1 lesions in the superior parietal and superior frontal regions predicted depression in MS patients [8].

Ordinary MRI tests could sensitively display MS lesions; however they lack pathological specifications [9]. Researchers have proved that the concealing injuries existed in MS patients while the outcomes of ordinary MRI displayed normal [10] (i.e., the micropathology alteration exists and its DTI data changes also) [11, 12]. DTI enables the random diffusional motion of water molecules to be measured, thus providing metrics such as mean diffusivity (MD) and FA in order to allow quantification of the size and geometry of water-filled spaces [13] and provide complete pathology message of brain tissues. In the past few years, DTI studies have been widely applied to the CNS of MS and optical neuromyelitis, and they have demonstrated a powerful means to early diagnosis and patient's condition monitoring.

Previous DTI studies generally adopted region of interest based analysis which needs specially prior information. Considering that MS may involve wide range of axonal degeneration, the whole brain analysis may be an optical method and lead to improved sensitivity and specificity to the disease and its related clinical impairments. Methods on the structural changes of WM fiber tracts in current research are mainly including hand-painted region of interest (ROI), voxel-based analysis (VBA), and TBSS, in which VBA is the most common research method. But for the anisotropic larger DTI image space, the registration accuracy is not high by VBA method which will lead to a certain difference based on the research results for the DTI images when different researchers are in view of the same kind of neurological diseases. However, TBSS analysis method aims at the main problem of image space registration rate, modifying from the registration rate algorithm, and makes the registration rate improved. So we chose TBSS as the analysis method. TBSS, adopting skeletonized processing ideas, projects individual fiber bundle FA value onto average FA bundle skeleton templates and accomplishes the justification of different subject fabric without taking standardization and smoothing [14] and thus significantly improves group comparison fidelity.

In the present study, we aimed at exploring global microstructural changes in MS and defining which changes are particularly affected by the disease such as the EDSS and HAMD scores and inquiring the relationships between FA values and HAMD as well as EDSS.

\section{Materials and Methods}

2.1. Subjects. In this study, we recruited the 15 patients who suffered from RRMS and were treated in Dongzhimen hospital in-patient or out-patient department from January 2012 to November 2013. All patients were in line with the revised McDonald criteria [15] and classification standard. The inclusion criteria were (1) participants were in the remission stage, who had no acute attack and did not have an exacerbation of their MS during the last month; (2) they were not currently taking any glucocorticosteroid medication; (3) their medication and treatment had no obvious adjustment recently and all of them had no history of serious psychiatric illness or neurologic disease other than MS; (4) Chinese was the primary language of all the participants; (5) the righthanded subjects according to the modified Edinburgh Handedness Questionnaire [16] were included. Those participants who had contraindications to MRI, poor quality of the images acquired, or showed one or more gadolinium-enhancing lesions (GEL) on baseline MRI were excluded to avoid effects of edema and inflammation on DTI measures [17]. Fifteen age- and sex-matched right-handed healthy subjects were used as a control group.

2.2. Clinical Assessments. For every participant, sex, age, onset age, disease duration, first onset symptoms, and recurrent symptoms variables were obtained while healthy controls' age and gender were also collected. A single neurologist assessed patients' disability using the EDSS [18] at the day of the neuropsychological assessment. All subjects were clinically evaluated by means of HAMD by themselves and two highly trained doctors on the same day prior to scanning. In this study, we used the HAMD 24 version and the criteria were as follows: total score $t<8$ was divided into normal, 8-20 might suffer from depression, 20-35 was a depressive patient certainly, and $>35$ suffered from the severe depression.

2.3. MRI Acquisition. In the study, high-resolution brain MRI was acquired by using 2 pulse sequences on a 3T Signa scanner (Verio, Siemens AG, Erlangen, Germany) with an 8 -channel head coil. The following sequences were acquired in a single session: (1) functional EPI oriented parallel to the AC-PC line and covering the whole brain to obtain sagittal sequence (repetition time $(\mathrm{TR})=2,000 \mathrm{~ms}$, echo time $(\mathrm{TE})=30 \mathrm{~ms}$, matrix $=64 \mathrm{~mm} \times 64 \mathrm{~mm}$, and field of view $(\mathrm{FOV})=225 \mathrm{~mm} \times 225 \mathrm{~mm}), 36$ slices, $3.5 \mathrm{~mm}$ thick, and no gap; (2) 3D magnetization-prepared rapid acquisition gradient echo (MPRAGE) sequence with 176 coronary, $1 \mathrm{~mm}$ slices, and $0 \mathrm{~mm}$ gap $(\mathrm{TR}=2,700 \mathrm{~ms}, \mathrm{TE}=$ $2.97 \mathrm{~ms}$, flip angle $=7^{\circ}$, matrix $=256 \mathrm{~mm} \times 256 \mathrm{~mm}, \mathrm{FOV}=$ $250 \mathrm{~mm} \times 250 \mathrm{~mm}$, and voxel size $=1 \mathrm{~mm} \times 1 \mathrm{~mm} \times 1 \mathrm{~mm}$ ); (3) T2-weighted image with a fast-spin echo sequence in the axial plane $(\mathrm{TR}=6,000 \mathrm{~ms}, \mathrm{TE}=94 \mathrm{~ms}$, matrix $=$ $320 \mathrm{~mm} \times 320 \mathrm{~mm}$, and FOV $=220 \mathrm{~mm} \times 220 \mathrm{~mm}), 25$ slices, $4 \mathrm{~mm}$ thick, and no gap; (4) T2-Flair weighted image $\left(\mathrm{TR}=8,800 \mathrm{~ms}, \mathrm{TE}=82 \mathrm{~ms}\right.$, flip angle $=150^{\circ}$, matrix $=$ $256 \mathrm{~mm} \times 256 \mathrm{~mm}$, and FOV $=240 \mathrm{~mm} \times 240 \mathrm{~mm})$. DTI data acquisition was acquired with an axial single-shot echo planar spin echo sequence with 30 gradient directions $(\mathrm{TR}=18,000 \mathrm{~ms}, \mathrm{TE}=94 \mathrm{~ms}$, matrix $=160 \mathrm{~mm} \times$ $160 \mathrm{~mm}, \mathrm{FOV}=256 \mathrm{~mm} \times 256 \mathrm{~mm}, b=0$ and 1,000), 80 slices, $1.5 \mathrm{~mm}$ thick, and no gap. Image data processing was performed on a Linux workstation using Jim 5.0 software (Xinapse System, Leicester, UK; http://www.xinapse.com/), the Functional MRI of the Brain (FMRIB) software library (FSL) 4.1 package (FMRIB Image Analysis Group, Oxford, UK; http://www.fmrib.ox.ac.uk/fsl/), MATLAB 7.0 (MathWorks, Natick, Massachusetts, USA), and the Statistical Parametric Mapping 8 (SPM8) software (Wellcome Department of Cognitive Neurology, London, UK; http://www.fil .ion.ucl.ac.uk/spm/). 
2.4. DTI Analysis. Diffusion data were preprocessed and analyzed using tools from the Oxford University Centre for FMRIB software library (FSL version 4.1). First, the b0 image of each subject was skull-stripped using the brain extraction tool. The data was corrected for subject motion and eddycurrent induced geometrical distortions, and the diffusion sensitizing gradients ("bvecs") were rotated to correct for motion. Using FDT, the diffusion tensor model was fit to the data, from which FA images were calculated.

For TBSS, all subjects' FA data was registered to a common space (the FA158 MNI space template) using a combination of affine and nonlinear registration. A mean FA image was created and eroded to a skeleton and threshold at FA $>0.25$. Each subject's aligned FA data was then projected onto this skeleton and the resulting alignment-invariant representation of the central trajectory of WM pathways was used for voxel-wise statistical analysis (Randomize, 5000 permutations). The contrast TBI < controls was examined using threshold free cluster enhancement (TFCE), with correction for multiple comparisons at $P<0.05$.

2.5. Statistical Analysis. The Statistical Package of Social Sciences (17.0; SPSS Inc., Chicago, IL) software was used to carry out the statistical analysis. All values were reported as mean \pm standard deviation (SD) or median (range) as appropriate. Differences between groups were tested using paired $t$-test for continuous and categorical variables, respectively. Correlations among global DTI measures and between global MRI values and clinical scores (EDSS and HAMD) were analyzed by univariate analysis (Pearson's correlation coefficient) after correction for age, and results corrected for multiple comparisons were needed.

\section{Results}

3.1. Demographic and Clinical Characteristics. In our study, fifteen patients ( 4 males and 11 females) were obtained according to the inclusion and exclusion criteria, who were aged from 19 to 57 years $(37.53 \pm 11.57)$. The disease duration of these patients ranged from 2 to 34 years $(7.27 \pm 8.55)$, onset age was from 13 to 53 years $(30 \pm 11.62)$, and the median EDSS score was 2 (range from 0 to 4 ). We also recruited 15 sex- and age-matched healthy volunteers who were aged from 23 to 60 years $(36.67 \pm 12.60)$ and had no history of physical illness or psychiatric disorder, and their nervous system examination was normal as the control group. A paired $t$-test was served to assess between-group differences of age between controls and patients $(P=0.407>0.05)$.

3.2. Clinical Rating Scales. In our research, patients and healthy controls (HC) were valued clinically by EDSS and HAMD. EDSS scores in patients ranged from 0 to $4(1.73 \pm$ 1.36), and in HC EDSS scores were all 0. HAMD scores in patients were from 1 to $35(12.80 \pm 11.620)$, while in HC they were from 0 to $14(5.07 \pm 3.918)$. Our study showed that there was a significant difference in HAMD score $(P=0.046<$ 0.05 ) between patients and HC. And among the 15 patients there were 9 people whose HAMD score was greater than or equal to 8 , accounting for $60 \%$.
3.3. TBSS Analysis. Quantitative comparison for TBSS analysis demonstrated widespread statistically significant differences in FA values $(P<0.05$, corrected for multiple comparisons), and FA values in all patients were lower compared with control subjects. Areas of reduced FA were seen widely in the GM and WM, such as the frontal lobe, limbic system, occipital lobe, temporal lobe, and parietal lobe. In particular, the main differences were located in bilateral corpus callosum, inferior parietal lobule, precentral gyrus, postcentral gyrus, superior frontal gyrus, cingulate gyrus, cerebellar lingual, declive, culmen, fastigium, dentate nucleus, parahippocampal gyrus, hippocampus, precuneus, basal ganglia, hypothalamus, insula, thalamus, fusiform gyrus, superior and transverse temporal gyrus, and the left middle temporal gyrus. Regional increases in the FA values of patients were not found. Compared with $\mathrm{HC}$, the main lesions of the reduced FA value which has statistical differences and voxel were listed in Tables 1 and 2.

3.4. Correlation between Diffusion Parameters (FA) and Clinical Scores. Significant correlations were found between FA and EDSS in some lesions of WM and GM: the right inferior parietal lobule of WM $(r=0.6307, P=0.0117)$, the left anterior cingulate $(r=-0.5505, P=0.0335)$, and hippocampus ( $r=-0.5143, P=0.0498)$ of GM.

We also found the significant correlations between FA and HAMD in some lesions of WM and GM: the right posterior middle cingulate gyrus $(r=0.6265, P=0.0124)$, hippocampus $(r=0.5742, P=0.0252)$, and the left hypothalamus ( $r=0.5357, P=0.0396)$ of $\mathrm{GM}$; the right precentral gyrus $(r=0.6575, P=0.0077)$, cingulate gyrus $(r=-0.5959, P=0.091)$, and posterior cingulate $(r=$ $0.5742, P=0.0258)$ of WM.

$R$ statistics (http://www.r-project.org/) analysis providing Spearman correlation coefficients values and their statistical significance were reported in Table 3 and shown in Figures 1 and 2.

\section{Discussion}

In our work, the patients' onset age was from 13 to 53 years and the sex ratio (female to male) was 2.75 . This result suggested that MS tends to appear in the young and middle aged females which was consistent with most of the previous studies [19, 20]. The statistical difference of HAMD score $(P=0.046<$ 0.05 ) between patients and HC was obvious, and among the 15 patients there were 9 people whose HAMD score was greater than or equal to 8 , accounting for $60 \%$. This result indicated that MS patients had a high incidence of depressive symptoms which was supported by the previous literature study [21].

DTI, as a new technology, which developed on the basis of diffusion weighted imaging (DWI) and could display brain WM fiber bundle and its direction in vivo noninvasively, is mainly used to evaluate the structural integrity of the microstructure, water molecules isotropic and anisotropic diffusion movement, and so forth. The results of DTI manifested the damage lesions mainly by the measures of FA values 
TABLE 1: The areas of FA value significantly reduced in GM by TBSS analysis.

\begin{tabular}{|c|c|c|c|c|c|c|}
\hline & & & lairac & & & $V \mathrm{~mm}^{3}$ \\
\hline & & $X$ & $Y$ & $Z$ & $t$ value & $\mathrm{mm}$ \\
\hline Limbic system & & & & & & \\
\hline ACG & $\mathrm{L}$ & -3 & 31 & 15 & 3.846 & 65 \\
\hline BA24 & $\mathrm{R}$ & 3 & 26 & 19 & 5.182 & 159 \\
\hline ACG & $\mathrm{L}$ & -3 & 4 & -6 & 3.543 & 44 \\
\hline BA25 & $\mathrm{R}$ & 7 & 16 & -8 & 3.238 & 45 \\
\hline PCG & $\mathrm{L}$ & 14 & -50 & 8 & 3.135 & 122 \\
\hline BA29/30 & $\mathrm{R}$ & -5 & -60 & 9 & 2.002 & 39 \\
\hline MCG & $\mathrm{L}$ & -20 & -16 & 38 & 2.999 & 113 \\
\hline BA24 & $\mathrm{R}$ & 18 & -3 & 45 & 4.299 & 250 \\
\hline PMCG & $\mathrm{L}$ & -18 & -43 & 28 & 3.307 & 62 \\
\hline BA31 & $\mathrm{R}$ & 19 & -43 & 37 & 4.761 & 102 \\
\hline Amvodala & $\mathrm{L}$ & -19 & -6 & -19 & 3.355 & 48 \\
\hline Minyg guald & $\mathrm{R}$ & 19 & -6 & -18 & 2.113 & 81 \\
\hline Hinnocampus & $\mathrm{L}$ & -30 & -36 & -6 & 2.948 & 35 \\
\hline пірросапри & $\mathrm{R}$ & 33 & -14 & -18 & 3.682 & 97 \\
\hline Subcortical & & & & & & \\
\hline Putamen & $\mathrm{L}$ & -22 & -9 & 9 & 4.632 & 217 \\
\hline & $\mathrm{R}$ & 23 & -7 & 11 & 3.981 & 200 \\
\hline Thalamus & $\mathrm{L}$ & -5 & -9 & 13 & 3.947 & 143 \\
\hline Cerebellum & & & & & & \\
\hline CI & $\mathrm{L}$ & -4 & -46 & -15 & 4.041 & 60 \\
\hline CL & $\mathrm{R}$ & 3 & -48 & -18 & 2.968 & 32 \\
\hline Culmen & $\mathrm{L}$ & -1 & -54 & -14 & 4.073 & 1204 \\
\hline Canintio & $\mathrm{R}$ & 14 & -41 & -18 & 4.317 & 894 \\
\hline Declive & $\mathrm{L}$ & -1 & -55 & -14 & 3.875 & 363 \\
\hline & $\mathrm{R}$ & 3 & -55 & -11 & 4.160 & 152 \\
\hline
\end{tabular}

and decreased FA values could indicate a result of demyelination processes [22]. In the present study, we found that all the patients presented attenuated FA values when compared with HC. Similar results could be seen in some recent studies $[23,24]$. Our data also confirmed that abnormalities in all the patients involved both WM and GM damage, which was consistent with many researches $[13,25,26]$. And our imaging data indicated that the lesions in WM of patients were mainly located in bilateral frontal lobe, limbic lobe, parietal lobe, occipital lobe, temporal lobe, corpus callosum, and sublobar while in GM the numerous lesions were primarily located in the limbic, sublobar, and cerebellum. In some previous researches, lesion was seen not only in the neocortex (especially in the cingulate cortex) $[25,27]$ but also in the GM of the thalamus, hypothalamus, cerebellum [27], basal ganglia [27, 28], and hippocampus [27, 29]. Audoin et al. [30] have reported that GM atrophy is associated with the bilateral insula, orbitofrontal cortices, internal and inferior temporal regions, thalamus, caudate nuclei, lenticular nuclei, cerebellum, and the posterior cingulate cortex. According to our result, it was roughly consistent with these previous studies.

However, most previous works adopted regional analysis, studying only certain parts of the brain, such as the normal appearing WM (NAWM) and GM (NAGM) [31], or the cerebellum [32], thalamus [33], and corpus callosum [34, 35]. Different from these reports, we studied WM and GM of the whole brain to find out the lesions by using a relatively new analysis method-TBSS. Hence, our research showed wider range of the lesions and this study could more fully state the distribution characteristics of the lesion site in the patients' brain DTI.

In this work, we adopted HAMD as evaluation index of psychological function and the relationships between FA value of the lesions in WM and GM of the entire brain and the HAMD scores in patients were explored. We investigated that HAMD scores were positively correlated with FA values in the left hypothalamus, right posterior middle cingulate gyrus and hippocampus of GM, the right precentral gyrus, and posterior cingulate of WM. These results told us that depressive symptoms were mainly negatively associated with the degree of demyelinating lesions in limbic system and frontal lobe, which had been reported in the previous paper [36]. Gobbi et al. [37] reported that depression in MS is linked to the atrophy of cortical regions located in the bilateral frontal lobes. Feinstein et al. [31] found that depressed subjects had a higher hypointense lesion volume in the right medial inferior frontal region, while having a smaller NAWM 
TABLE 2: The areas of FA value significantly reduced in WM by TBSS analysis.

\begin{tabular}{|c|c|c|c|c|c|c|}
\hline & & \multicolumn{3}{|c|}{ Talairach } & \multirow{2}{*}{$t$ value } & \multirow{2}{*}{$V \mathrm{~mm}^{3}$} \\
\hline & & $X$ & $Y$ & $Z$ & & \\
\hline \multicolumn{7}{|l|}{ Limbic system } \\
\hline ACG & $\mathrm{L}$ & -7 & 24 & -4 & 4.655 & 158 \\
\hline BA24 & $\mathrm{R}$ & 15 & 18 & 23 & 4.275 & 318 \\
\hline \multirow{2}{*}{ CG } & $\mathrm{L}$ & -15 & -33 & 35 & 5.154 & 1561 \\
\hline & $\mathrm{R}$ & 19 & -28 & 34 & 4.884 & 1798 \\
\hline PHG & $\mathrm{L}$ & -22 & -18 & -13 & 4.989 & 663 \\
\hline BA28/36 & $\mathrm{R}$ & 19 & -41 & 2 & 5.884 & 654 \\
\hline PCG & $\mathrm{L}$ & -11 & -56 & 6 & 2.974 & 125 \\
\hline BA29/30 & $\mathrm{R}$ & 14 & -53 & 14 & 4.149 & 253 \\
\hline \multicolumn{7}{|l|}{ Subcortical } \\
\hline \multirow{3}{*}{$\mathrm{CC}$} & $\mathrm{IH}$ & -1 & -16 & 24 & 2.889 & 63 \\
\hline & $\mathrm{L}$ & -3 & 13 & 20 & 3.917 & 1180 \\
\hline & $\mathrm{R}$ & 13 & -29 & 25 & 6.924 & 1068 \\
\hline Insula & $\mathrm{L}$ & -38 & -43 & 20 & 3.899 & 123 \\
\hline BA13 & $\mathrm{R}$ & 39 & -41 & 19 & 4.562 & 107 \\
\hline \multicolumn{7}{|l|}{ Frontal lobe } \\
\hline CG & $\mathrm{L}$ & -17 & 15 & 36 & 3.727 & 38 \\
\hline $\operatorname{PrG}$ & $\mathrm{L}$ & -25 & -17 & 50 & 2.299 & 30 \\
\hline BA4 & $\mathrm{R}$ & 37 & -10 & 26 & 2.836 & 55 \\
\hline \multirow{2}{*}{$\mathrm{CC}$} & $\mathrm{L}$ & -11 & 20 & 18 & 3.835 & 207 \\
\hline & $\mathrm{R}$ & 13 & 19 & 20 & 3.922 & 146 \\
\hline \multicolumn{7}{|l|}{ Parietal } \\
\hline \multirow{2}{*}{ IPL } & $\mathrm{L}$ & -42 & -42 & 25 & 3.559 & 135 \\
\hline & $\mathrm{R}$ & 38 & -42 & 26 & 4.286 & 110 \\
\hline Precuneus & $\mathrm{L}$ & -21 & -59 & 32 & 4.402 & 435 \\
\hline BA31 & $\mathrm{R}$ & 19 & -42 & 44 & 5.347 & 271 \\
\hline
\end{tabular}

BA: Brodmann area; ACG: anterior cingulate gyrus; CG: cingulate gyrus; PCG: posterior cingulate gyrus; MCG: middle cingulate gyrus; PMCG: posterior middle cingulate gyrus; CC: corpus callosum; IPL: inferior parietal lobule; PrG: precentral gyrus; DLPFC: dorsolateral prefrontal cortex; AG: angular gyrus; CL: cerebellar lingual; IH: interhemispheric; PHG: parahippocampal gyrus.

TABLE 3: Significant correlations (Spearman correlation coefficients) between diffusion parameters (FA) and clinical scores.

\begin{tabular}{llll}
\hline \multicolumn{1}{c}{ CRS and correlated lesions } & & $R$ value & $P$ value \\
\hline EDSS (GM) & & & \\
ACC & $\mathrm{L}$ & $-0.5505^{*}$ & 0.0335 \\
Hippocampus & $\mathrm{L}$ & $-0.5143^{*}$ & 0.0498 \\
EDSS (WM) & & & $0.6307^{*}$ \\
$\quad$ IPL & & & 0.0117 \\
HAMD (GM) & $\mathrm{R}$ & $0.6265^{*}$ & 0.0124 \\
pMCC & $\mathrm{R}$ & $0.5742^{*}$ & 0.0252 \\
Hippocampus & $\mathrm{L}$ & $0.5357^{*}$ & 0.0396 \\
Hypothalamus & & & \\
HAMD (WM) & $\mathrm{R}$ & $0.6575^{*}$ & 0.0077 \\
PrG & $\mathrm{R}$ & -0.5959 & 0.0910 \\
CG & $\mathrm{R}$ & $0.5724^{*}$ & 0.0258 \\
PCC & & & \\
\hline
\end{tabular}

${ }^{*}$ Significant correlation, $P<0.05$.

R: right, L: left, CRS: clinical rating scales, ACC: anterior cingulate cortex, IPL: inferior parietal lobule, pMCC: posterior middle cingulate cortex, PrG: precentral gyrus, CG: cingulate gyrus, and PCC: posterior cingulate cortex. 


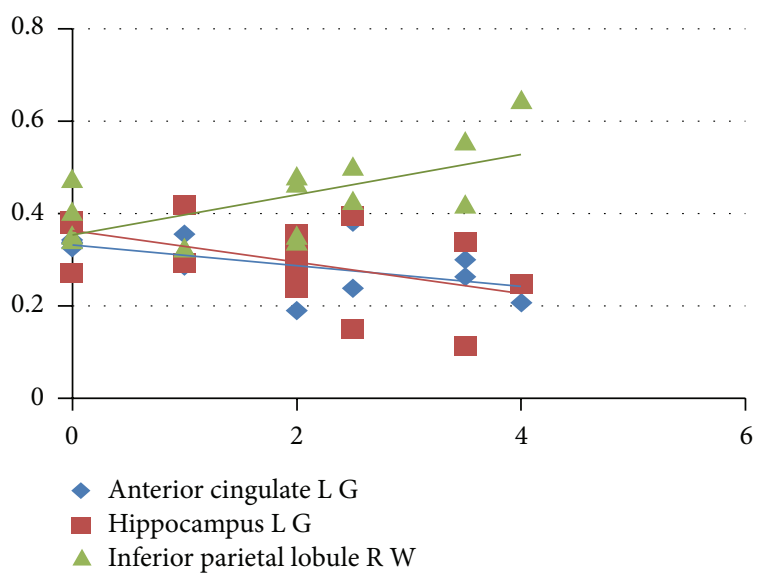

FIGURE 1: The correlation between EDSS and MRI lesion in GM and WM.

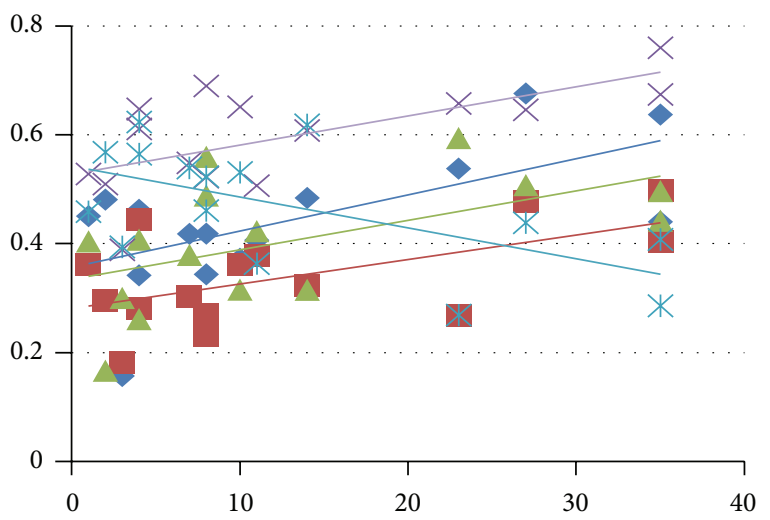

- Posterior middle cingulate gyrus R G

- Hippocampus R G

A Hypothalamus L G

$\times$ Precentral gyrus R W

* Posterior cingulate $\mathrm{R} \mathrm{W}$

FIGURE 2: The correlation between HAMD and MRI lesion in GM and WM. R: right, L: left, G: GM, and W: WM.

volume in the left superior frontal region and lower FA in the left anterior temporal NAWM and NAGM regions, respectively. The cause may be that frontal lobe and limbic system are relevant to human's affect, memory, and learning. Once these functions defected, depression would occur in patients. Besides, we also found the negative correlations between FA and HAMD in cingulate gyrus, which was never found before. The mechanism is unknown, and the ongoing compensatory cerebral process at work in the MS brain attempting to maintain an euthymic state which was found by functional MRI (fMRI) [38] may be correlated with it.

EDSS is regarded as the evaluation index of the neurologic deficits and many papers have studied the relationship between FA value of the lesions and EDSS score. Some suggested that there was no correlation $[33,39,40]$, while some showed that there was a positive correlation between them: EDSS was positively correlated with FA value of the normal appear thalamus $(r=0.66, P=0.045)$ [41], caudate $(r=0.444, P<0.01)$, and thalamus $(r=0.362, P<0.05)$ [42] and was slightly negatively correlated with the atrophy of the right cerebellum $(r=-0.37, P=0.0027)$ [30]. However, one paper showed that it was strongly negatively associated with some lesions $(r=-0.82, P=0.013)$ [23]. But the cause of the negative correlation between EDSS and the degree of demyelination still needs further investigations. For the pathological changes in this relationship, according to Tedeschi et al. [43] and Hofstetter et al. [44], GM atrophy is associated with MS clinical disability. Routinely detectable cortical lesions are related to physical disability [45]. And Ciccarelli et al. [46] have reported that, in patients with RRMS, there was a strong correlation between EDSS score and FA in both supratentorial and infratentorial NAWM. Gorgoraptis et al. [39] found that smaller paracentral cortex volume was associated with worse walking ability, as measured by the TWT. One research showed that either the NAWM FA or the GM volume in each of these regions correlated with disability $[47,48]$. Studies from DTI features of RRMS patients show that GM atrophy is a better indicator of disability progression than WM atrophy or accumulation of lesion burden [49-52]. In our study, EDSS is strongly correlated with FA value in the right inferior parietal lobule of WM positively and the left anterior cingulate and hippocampus of GM negatively in patients. Anterior cingulate cortex (ACC), including Brodmann 24, 25, and 32 area, which is located in the medial area of frontal lobe, can monitor the ongoing goal orientation behavior, provide signals in response to conflict or mistakes, and allocate the attentive resources effectively in related brain regions according to the requirements of the current task processing, and therefore it may be a senior regulatory structure in the executive function neural network [53], while hippocampus is responsible for learning and memory. Considering the functions of the damage regions in human, we all agreed that once these functions disappear this may lead to clinical disability in patients with MS.

However, our study has some limitations. First, this research only recruited the MS patients who were in remission and did not include patients who were in the acute stage as a control group for the related pathology study. Second, we conducted a cross-sectional study only, not for long-time follow-up observations. Third, in our work some appearances cannot be explained just with our present knowledge and findings. For instance, EDSS was negatively correlated with the degree of demyelination in the right inferior parietal lobule of WM and the further causes of the positive relationship between HAMD and FA in the lesions. For further study, we will expand the scope of the study population, conduct longitudinal observation on the basis of the study, and further analyze the relationships between the lesions and clinical scores.

\section{Conclusions}

In conclusion, our study used TBSS analysis method for the whole brain DTI of RRMS patients who were in remission and a large amount of information was provided for multiple areas of the brain GM and WM in pathological changes. The characteristics of the various lesion areas and 
the relationships between the clinical scores of MS patients were discussed in this paper, providing the possible mechanisms for the pathogenesis of MS. In addition, we had shown that GM damage could explain clinical depression and disability better than WM. These findings were important for our understanding of MS and for future clinical trial design. And TBSS can be useful in future studies with other MS patient samples to provide an unbiased localization of damage and generate location-specific hypotheses.

\section{Conflict of Interests}

There are no competing interests.

\section{Authors' Contribution}

Yujuan Shen and Fangyuan Cui contributed equally to this work.

\section{Acknowledgments}

This study was supported by the National Natural Science Foundation of China (Grant no. 81071217, no. 81371630), the Fundamental Research Funds for the Central Universities, the Beijing Nova Program (Grant no. Z111101054511116), 2011 Beijing University of Chinese Medicine subject project independently (Grant no. 2011JYBZZJS-014), and the Beijing Natural Science Foundation (Grant no. 4122082).

\section{References}

[1] E. M. Frohman, M. K. Racke, and C. S. Raine, "Medical progress: multiple sclerosis-the plaque and its pathogenesis," New England Journal of Medicine, vol. 354, no. 9, pp. 942-955, 2006.

[2] M. Pugliatti, S. Sotgiu, and G. Rosati, "The worldwide prevalence of multiple sclerosis," Clinical Neurology and Neurosurgery, vol. 104, no. 3, pp. 182-191, 2002.

[3] A. Feinstein, "Multiple sclerosis and depression," Multiple Sclerosis, vol. 17, no. 11, pp. 1276-1281, 2011.

[4] M. Coenen, B. Basedow-Rajwich, N. König, J. Kesselring, and A. Cieza, "Functioning and disability in multiple sclerosis from the patient perspective," Chronic Illness, vol. 7, no. 4, pp. 291-310, 2011.

[5] R. Bakshi, D. Czarnecki, Z. A. Shaikh et al., "Brain MRI lesions and atrophy are related to depression in multiple sclerosis," NeuroReport, vol. 11, no. 6, pp. 1153-1158, 2000.

[6] A. Feinstein, "Neuropsychiatric syndromes associated with multiple sclerosis," Journal of Neurology, vol. 254, no. 2, pp. 7376, 2007.

[7] J. Pujol, J. Bello, J. Deus, J. L. Marti-Vilalta, and A. Capdevila, "Lesions in the left arcuate fasciculus region and depressive symptoms in multiple sclerosis," Neurology, vol. 49, no. 4, pp. 1105-1110, 1997.

[8] M. Zorzon, R. de Masi, D. Nasuelli et al., "Depression and anxiety in multiple sclerosis. A clinical and MRI study in 95 subjects," Journal of Neurology, vol. 248, no. 5, pp. 416-421, 2001.

[9] Y. Miki, R. I. Grossman, J. K. Udupa et al., "Relapsing-remitting multiple sclerosis: longitudinal analysis of MR images_lack of correlation between changes in T2 lesion volume and clinical findings," Radiology, vol. 213, no. 2, pp. 395-399, 1999.

[10] Y. Ge, R. I. Grossman, J. K. Udupa, J. S. Babb, L. J. Mannon, and J. C. McGowan, "Magnetization transfer ratio histogram analysis of normal-appearing gray matter and normal-appearing white matter in multiple sclerosis," Journal of Computer Assisted Tomography, vol. 26, no. 1, pp. 62-68, 2002.

[11] W. Rashid, A. Hadjiprocopis, C. M. Griffin et al., "Diffusion tensor imaging of early relapsing-remitting multiple sclerosis with histogram analysis using automated segmentation and brain volume correction," Multiple Sclerosis, vol. 10, no. 1, pp. 9-15, 2004.

[12] S. D. Roosendaal, J. J. G. Geurts, H. Vrenken et al., "Regional DTI differences in multiple sclerosis patients," NeuroImage, vol. 44, no. 4, pp. 1397-1403, 2009.

[13] M. Rovaris, F. Agosta, E. Pagani, and M. Filippi, "Diffusion tensor MR imaging," Neuroimaging Clinics of North America, vol. 19, no. 1, pp. 37-43, 2009.

[14] D. K. Jones, X. A. Chitnis, D. Job et al., "What happens when nine different group analyze the same DT-MRI data set using voxel-based methods," Proceedings of the International Society for Magnetic Resonance in Medicin, vol. 15, article 74, 2007.

[15] C. H. Polman, S. C. Reingold, and G. Edan, "Diagnostic criteria for multiple sclerosis: 2005 revisions to the 'McDonald Criteria", Annals of Neurology, vol. 58, pp. 840-846, 2005.

[16] R. C. Oldfield, "The assessment and analysis of handedness: the Edinburgh inventory," Neuropsychologia, vol. 9, no. 1, pp. 97-113, 1971.

[17] E. Sbardella, N. Petsas, F. Tona et al., "Assessing the correlation between grey and white matter damage with motor and cognitive impairment in multiple sclerosis patients," PLOS ONE, vol. 8, Article ID e63250, 2013.

[18] J. F. Kurtzke, "Rating neurologic impairment in multiple sclerosis: an expanded disability status scale (EDSS)," Neurology, vol. 33, no. 11, pp. 1444-1452, 1983.

[19] K. Jefferies, "The neuropsychiatry of multiple sclerosis," Advances in Psychiatric Treatment, vol. 12, no. 3, pp. 214-220, 2006.

[20] B. Reipert, "Multiple sclerosis: a short review of the disease and its differences between men and women," Journal of Men's Health and Gender, vol. 1, no. 4, pp. 334-340, 2004.

[21] K. Gottberg, U. Einarsson, S. Fredrikson, L. Von Koch, and L. W. Holmqvist, "A population-based study of depressive symptoms in multiple sclerosis in Stockholm county: association with functioning and sense of coherence," Journal of Neurology, Neurosurgery and Psychiatry, vol. 78, no. 1, pp. 60-65, 2007.

[22] S.-K. Song, S.-W. Sun, W.-K. Ju, S.-J. Lin, A. H. Cross, and A. H. Neufeld, "Diffusion tensor imaging detects and differentiates axon and myelin degeneration in mouse optic nerve after retinal ischemia," NeuroImage, vol. 20, no. 3, pp. 1714-1722, 2003.

[23] M. Onu, A. Roceanu, U. Sboto-Frankenstein et al., "Diffusion abnormality maps in demyelinating disease: correlations with clinical scores," European Journal of Radiology, vol. 81, no. 3, pp. e386-e391, 2012.

[24] K. Erb, G. Bohner, L. Harms et al., "Olfactory function in patients with multiple sclerosis: a diffusion tensor imaging study," Journal of the Neurological Sciences, vol. 316, no. 1-2, pp. 56-60, 2012. 
[25] M. Filippi and M. A. Rocca, "MR imaging of gray matter involvement in multiple sclerosis: implications for understanding disease pathophysiology and monitoring treatment efficacy," American Journal of Neuroradiology, vol. 31, no. 7, pp. 1171$1177,2010$.

[26] A. Kutzelnigg, C. F. Lucchinetti, C. Stadelmann et al., "Cortical demyelination and diffuse white matter injury in multiple sclerosis," Brain, vol. 128, no. 11, pp. 2705-2712, 2005.

[27] J. J. Geurts and F. Barkhof, "Grey matter pathology in multiple sclerosis," The Lancet Neurology, vol. 7, no. 9, pp. 841-851, 2008.

[28] I. E. C. Ormerod, D. H. Miller, W. I. McDonald et al., "The role of NMR imaging in the assessment of multiple sclerosis and isolated neurological lesions. A quantitative study," Brain, vol. 110, no. 6, pp. 1579-1616, 1987.

[29] S. D. Roosendaal, B. Moraal, H. Vrenken et al., "In vivo MR imaging of hippocampal lesions in multiple sclerosis," Journal of Magnetic Resonance Imaging, vol. 27, no. 4, pp. 726-731, 2008.

[30] B. Audoin, W. Zaaraoui, F. Reuter et al., "Atrophy mainly affects the limbic system and the deep grey matter at the first stage of multiple sclerosis," Journal of Neurology, Neurosurgery and Psychiatry, vol. 81, no. 6, pp. 690-695, 2010.

[31] A. Feinstein, P. O'Connor, N. Akbar, L. Moradzadeh, C. J. M. Scott, and N. J. Lobaugh, "Diffusion tensor imaging abnormalities in depressed multiple sclerosis patients," Multiple Sclerosis, vol. 16, no. 2, pp. 189-196, 2010.

[32] V. M. Anderson, C. A. M. Wheeler-Kingshott, K. Abdel-Aziz et al., "A comprehensive assessment of cerebellar damage in multiple sclerosis using diffusion tractography and volumetric analysis," Multiple Sclerosis, vol. 17, no. 9, pp. 1079-1087, 2011.

[33] F. Tovar-Moll, I. E. Evangelou, A. W. Chiu et al., "Thalamic involvement and its impact on clinical disability in patients with multiple sclerosis: a diffusion tensor imaging study at 3T," American Journal of Neuroradiology, vol. 30, no. 7, pp. 13801386, 2009.

[34] Y. Zhou, M. P. Milham, X.-N. Zuo et al., "Functional homotopic changes in multiple sclerosis with resting-state functional MR imaging," American Journal of Neuroradiology, vol. 34, pp. 11801187, 2013.

[35] T. Sigal, M. Shmuel, D. Mark, H. Gil, and A. Anat, "Diffusion tensor imaging of corpus callosum integrity in multiple sclerosis: correlation with disease variables," Journal of Neuroimaging, vol. 22, no. 1, pp. 33-37, 2012.

[36] P. V. Rabins, B. R. Brooks, and P. O’Donnell, "Structural brain correlates of emotional disorder in multiple sclerosis," Brain, vol. 109, no. 4, pp. 585-597, 1986.

[37] C. Gobbi, M. Rocca, and G. Riccitelli, "Influence of the topography of brain damage on depression and fatigue in patients with multiple sclerosis," Multiple Sclerosis, vol. 20, no. 2, pp. 192-201.

[38] L. Passamonti, A. Cerasa, M. Liguori et al., "Neurobiological mechanisms underlying emotional processing in relapsingremitting multiple sclerosis," Brain, vol. 132, no. 12, pp. 33803391, 2009.

[39] N. Gorgoraptis, C. A. M. Wheeler-Kingshott, T. M. Jenkins et al., "Combining tractography and cortical measures to test system-specific hypotheses in multiple sclerosis," Multiple Sclerosis, vol. 16, no. 5, pp. 555-565, 2010.

[40] S. Temel, H. D. Kekligkoglu, G. Vural et al., "Diffusion tensor magnetic resonance imaging in patients with multiple sclerosis and its relationship with disability," Neuroradiology Journal, vol. 26, pp. 3-17, 2013.
[41] K. M. Hasan, I. S. Walimuni, H. Abid et al., "Multimodal quantitative magnetic resonance imaging of thalamic development and aging across the human lifespan: implications to neurodegeneration in multiple sclerosis," Journal of Neuroscience, vol. 31, no. 46, pp. 16826-16832, 2011.

[42] S. Hannoun, F. Durand-Dubief, C. Confavreux et al., "Diffusion tensor-MRI evidence for extra-axonal neuronal degeneration in caudate and thalamic nuclei of patients with multiple sclerosis," American Journal of Neuroradiology, vol. 8, pp. 1363-1368, 2012.

[43] G. Tedeschi, L. Lavorgna, P. Russo et al., "Brain atrophy and lesion load in a large population of patients with multiple sclerosis," Neurology, vol. 65, no. 2, pp. 280-285, 2005.

[44] L. Hofstetter, Y. Naegelin, L. Filli et al., "Progression in disability and regional grey matter atrophy in Relapsing-remitting multiple sclerosis," Multiple Sclerosis, 2013.

[45] A. Mike, B. I. Glanz, P. Hildenbrand et al., "Identification and clinical impact of multiple sclerosis cortical lesions as assessed by routine 3T MR imaging," American Journal of Neuroradiology, vol. 32, no. 3, pp. 515-521, 2011.

[46] O. Ciccarelli, D. J. Werring, C. A. M. Wheeler-Kingshott et al., "Investigation of MS normal-appearing brain using diffusion tensor MRI with clinical correlations," Neurology, vol. 56, no. 7, pp. 926-933, 2001.

[47] L. V. Zollinger, T. H. Kim, K. Hill, E. K. Jeong, and J. W. Rose, "Using diffusion tensor imaging and immunofluorescent assay to evaluate the pathology of multiple sclerosis," Journal of Magnetic Resonance Imaging, vol. 33, no. 3, pp. 557-564, 2011.

[48] A. Giorgio, J. Palace, H. Johansen-Berg et al., "Relationships of brain white matter microstructure with clinical and MR measures in relapsing-remitting multiple sclerosis," Journal of Magnetic Resonance Imaging, vol. 31, no. 2, pp. 309-316, 2010.

[49] E. Fisher, J.-C. Lee, K. Nakamura, and R. A. Rudick, "Gray matter atrophy in multiple sclerosis: a longitudinal study," Annals of Neurology, vol. 64, no. 3, pp. 255-265, 2008.

[50] L. K. Fisniku, D. T. Chard, J. S. Jackson et al., "Gray matter atrophy is related to long-term disability in multiple sclerosis," Annals of Neurology, vol. 64, no. 3, pp. 247-254, 2008.

[51] D. Horakova, J. L. Cox, E. Havrdova et al., "Evolution of different MRI measures in patients with active relapsing-remitting multiple sclerosis over 2 and 5 years: a case-control study," Journal of Neurology, Neurosurgery and Psychiatry, vol. 79, no. 4, pp. 407414, 2008.

[52] G. Tedeschi, D. Dinacci, M. Comerci et al., "Brain atrophy evolution and lesion load accrual in multiple sclerosis: a 2-year follow-up study," Multiple Sclerosis, vol. 15, no. 2, pp. 204-211, 2009.

[53] M. S. Gazzaniga, R. B. Ivry, and G. R. Mangun, Cognitive Neuroscience: The Biology of the Mind, W. W. Norton \& Company, New York, NY, USA, 2nd edition, 2002. 


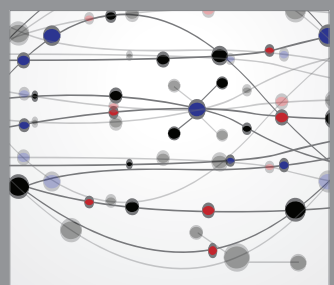

The Scientific World Journal
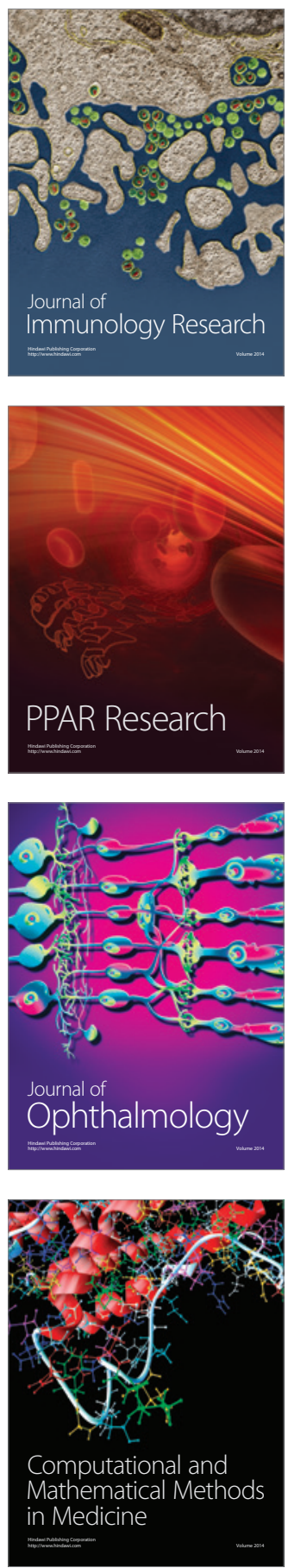

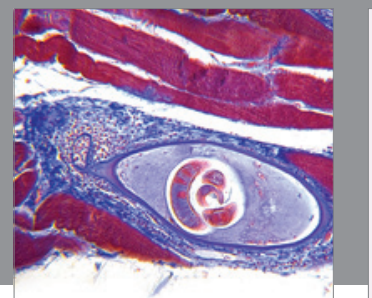

Gastroenterology

Research and Practice
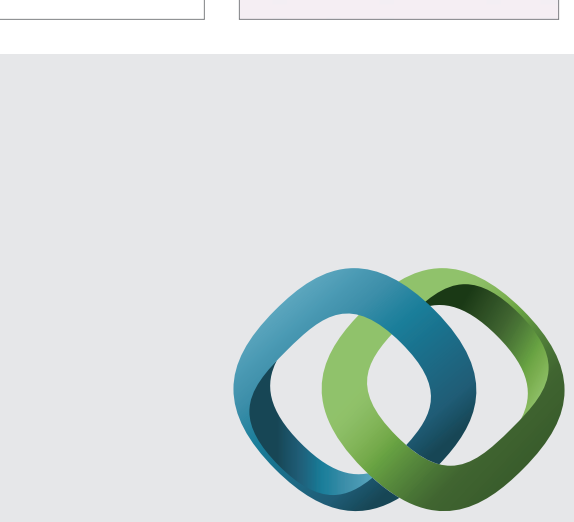

\section{Hindawi}

Submit your manuscripts at

http://www.hindawi.com
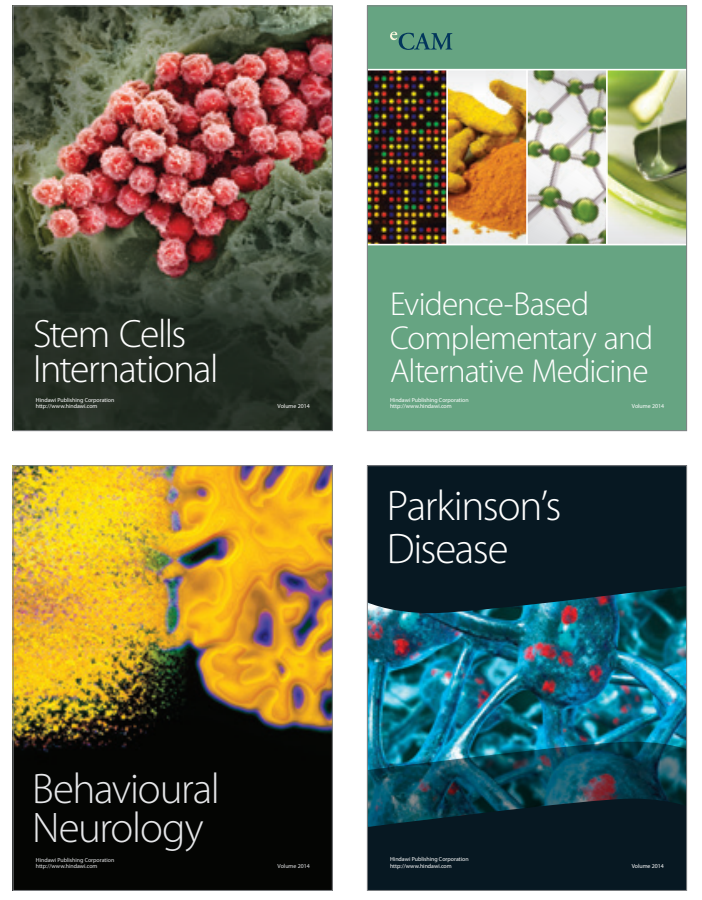
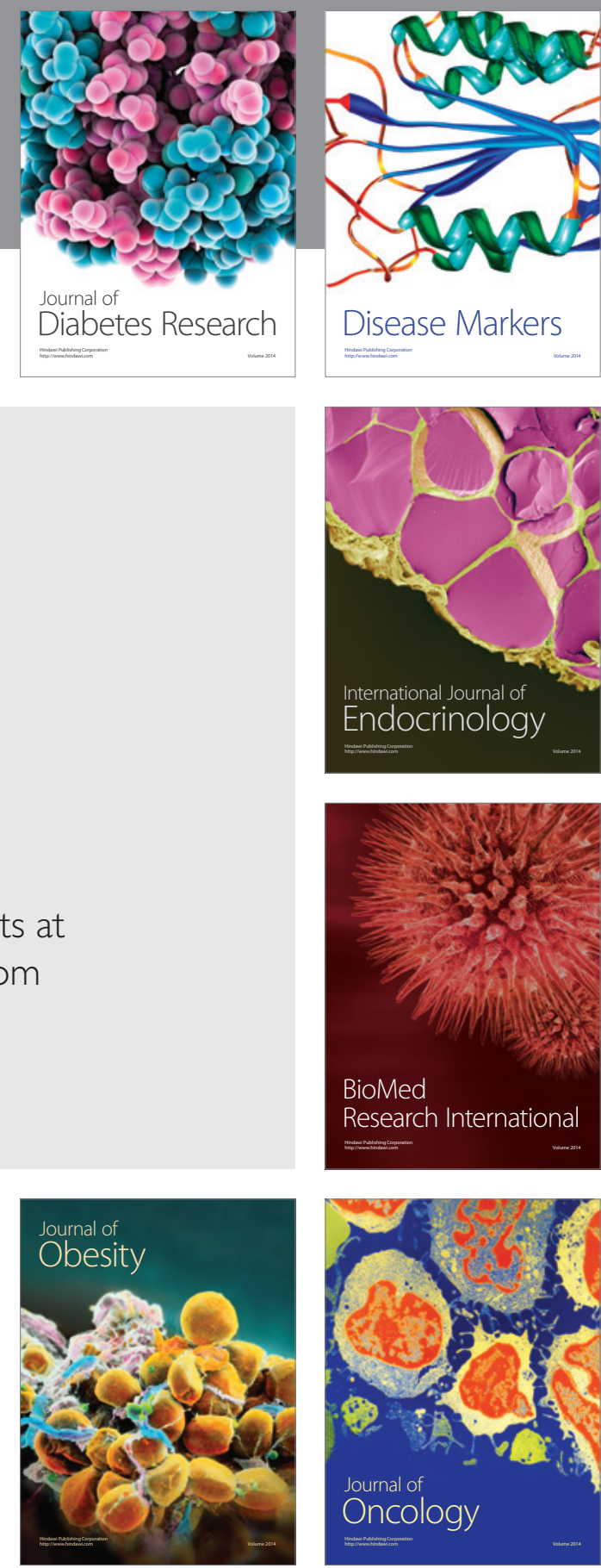

Disease Markers
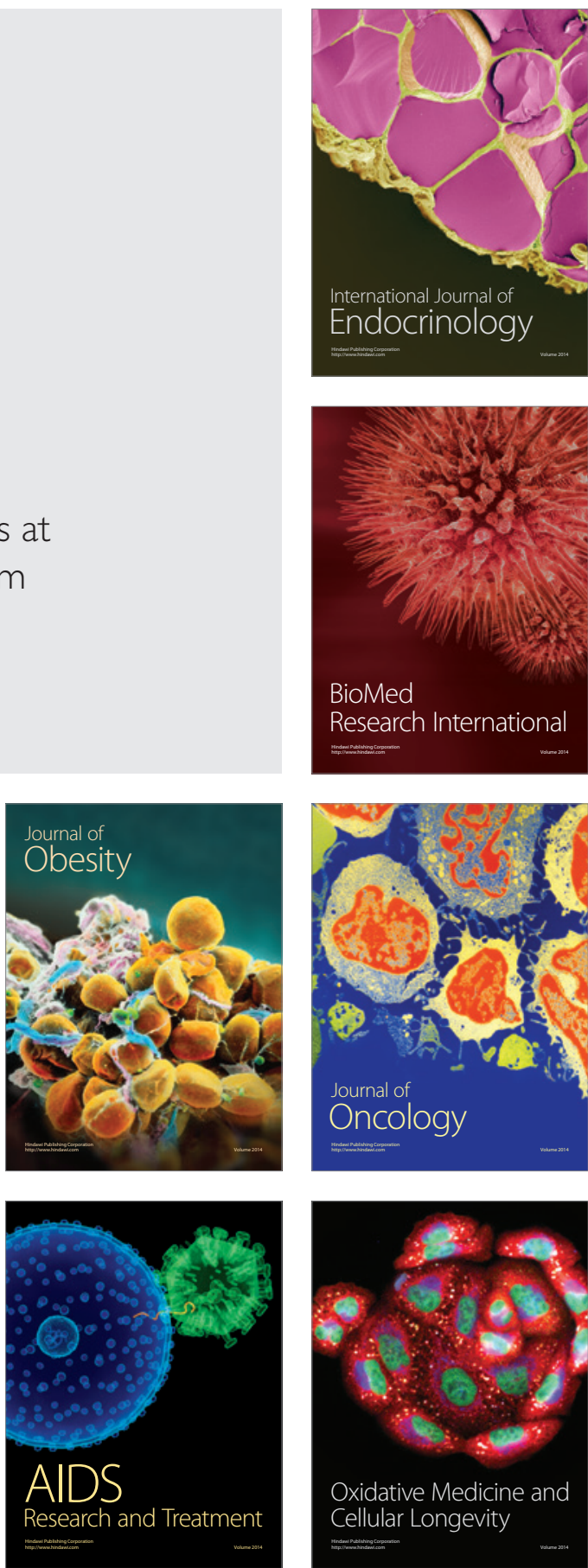